\title{
Vessel Size Imaging is Associated with IDH Mutation and Patient Survival in Diffuse Lower-Grade Glioma
}

This article was published in the following Dove Press journal: Cancer Management and Research

\author{
Houyi Kang ${ }^{1,2, *}$ \\ Peng Chen ${ }^{1,2, *}$ \\ Hong Guo ${ }^{1,2}$ \\ Letian Zhang ${ }^{1,2}$ \\ Yong $\operatorname{Tan}^{1,2}$ \\ Hualiang $\mathrm{Xiao}^{3}$ \\ Ao Yang ${ }^{4}$ \\ Jingqin Fang ${ }^{1,2}$ \\ Weiguo Zhang ${ }^{1,2}$
}

'Department of Radiology, Daping Hospital, Army Medical University, Chongqing 400042, People's Republic of China; ${ }^{2}$ Chongqing Clinical Research Center of Imaging and Nuclear Medicine, Chongqing, People's Republic of China; ${ }^{3}$ Department of Pathology, Daping Hospital, Army Medical University, Chongqing, People's Republic of China; ${ }^{4}$ Department of Traffic Injury Research Office, Daping Hospital, Army Medical Center of PLA, Chongqing, People's Republic of China

*These authors contributed equally to this work
Correspondence: Weiguo Zhang; Jingqin Fang

Email wgzhang0I@I63.com;

jingqin0405@163.com
Background: Patients with isocitrate dehydrogenase (IDH) mutant gliomas have better survival and appear to be more sensitive to chemotherapy than their IDH wild-type counterparts. We attempted to assess the correlations of vessel size imaging (VSI) values with IDH mutation status and patient survival in diffuse lower-grade glioma (LGG).

Methods: We enrolled 60 patients with diffuse LGGs, among which 43 had IDH-mutant tumors. All patients underwent VSI examination and VSI values for active tumors were calculated. Receiver operating characteristic (ROC) curves were established to evaluate the detection efficiency. Logistic regression was employed to determine the ability of variables to discriminate IDH mutational status. Kaplan-Meier survival analysis and Cox proportional hazards models were utilized to estimate the correlations of VSI values and other risk factors with patient survival.

Results: We observed that VSI values were lower in IDH-mutant LGGs than IDH wild-type LGGs. The VSImax and VSImean values had AUC values of 0.7305 and 0.7401 , respectively, in distinguishing IDH-mutant LGGs from IDH wild-type LGGs. Logistic regression showed that VSImean values, age and tumor location were associated with IDH-mutant status, and the formula integrating the three factors had an AUC value of 0.7798 when distinguishing IDH-mutant LGGs from IDH wild-type LGGs. Moreover, LGG patients with high VSI values exhibited worse survival rates than those with low VSI values for both progression-free survival (PFS) and overall survival (OS). Multivariate Cox proportional hazards regression analysis suggested that IDH mutation status, VSImean values and multiple lesions or lobes were risk factors for PFS of LGG patients.

Conclusion: VSI value is associated with IDH genotype and maybe an independent predictor of the survival of patients with LGGs.

Keywords: vessel size imaging, glioma, isocitrate dehydrogenase, IDH, mutation, survival

\section{Introduction}

Infiltrating astrocytoma is the most common type of primary brain tumor and can be divided into grades II, III and IV according to the 2007 World Health Organization (WHO) classification. ${ }^{1}$ Diffuse lower-grade glioma (LGG) is an infiltrative brain tumor consisting of WHO grade II and III neoplasms, which presents a more indolent course compared to glioblastoma (GBM, grade IV), ${ }^{2,3}$ and LGG is also divided into a series of molecular subtypes based on isocitrate dehydrogenase (IDH) and $1 \mathrm{p} / 19 \mathrm{q}$ co-deletion status according to the 2016 WHO classification. ${ }^{1-3}$

The prognosis of patients with glioma is related to several clinical factors, such as age, extent of tumor resection, and treatment modality, ${ }^{4}$ and correlates with 
molecular markers, such as IDH mutation, O6methylguanine-DNA methyltransferase promotor methylation, and $1 \mathrm{p} / 19 \mathrm{q}$ co-deletion. Notably, IDH mutation occurs in the majority of LGGs and has been considered a critical prognostic molecular marker for LGG. ${ }^{4}$ Patients with IDH-mutant LGGs have better outcomes and appear to be more sensitive to chemoradiotherapy than those with IDH wild-type LGGs. ${ }^{5,6}$ Therefore, identification of IDH genotype is beneficial for predicting the prognosis of LGG patients and for determining therapeutic strategies. Currently, however, determination of IDH genotype in LGGs requires invasive approaches, such as surgery or pathological biopsy, and then the use of an immunohistochemistry test, which may have an approximately $15 \%$ rate of missed diagnosis with the use of the antibody, R132H. ${ }^{7}$ Therefore, it is urgently important to explore a noninvasive method for evaluating IDH mutation status.

Traditional magnetic resonance imaging (MRI) is a noninvasive imaging technology that generates anatomical images but cannot reflect tumor metabolism, so it is difficult to identify the molecular classification of gliomas. Functional MRI can obtain functional information by visualizing cortical activity and shows a certain effect in the molecular classification of gliomas, especially MR spectral imaging (MRS) and MR perfusionweighted imaging (PWI). ${ }^{8,9}$ For example, MRS can reach 80-90\% accuracy in recognizing IDH mutation by detecting 2-hydroxyglutarate (2HG), a metabolite of IDH. ${ }^{8}$ However, this technology is challenging and difficult to apply in clinical practice. ${ }^{8}$ Cerebral blood volume (CBV) derived from PWI has been proposed to assess the prognosis of glioma patients and the molecular classification of glioma to some extent. ${ }^{1,10}$ Vessel size imaging (VSI), another MR perfusion technology, can describe the structural heterogeneity of brain microvasculature based on a gradient-echo (GE)/spin-echo (SE) echo planar imaging sequence. ${ }^{11}$ Our previous study indicated that VSI values are more accurate than relative $\mathrm{CBV}$ (rCBV) values in glioma grading, ${ }^{12}$ suggesting that VSI may reflect the degree of malignancy. However, the efficiency of VSI in diagnosing the IDH genotype and predicting the prognosis of patients with LGGs has not been fully elucidated.

In the present study, we evaluated the diagnostic performance of VSI in recognizing IDH mutation status in LGGs and predicting the survival rates of LGG patients.

\section{Materials and Methods}

\section{Patients}

A total of 60 patients who underwent initial surgery and were histopathologically diagnosed with grade II or III glioma in Army Medical Center from March 2013 to May 2016 were included in this study. The characteristics of all patients are shown in Table 1. All tissue samples were tested for IDH mutation status. The inclusion criteria include the following: 1) the lesion is located in the supratentorial space; 2 ) the tumor did not spread, and the patient was over 18 years old; 3) chemotherapy was not performed before glioma surgery, and a complete treatment regimen and radiotherapy dose records were required for patients with recurrence; 4) IDH mutation and 1p/19q codeletion status are known. Exclusion criteria include the following: 1) patient with other brain tumors, meningoencephalitis or tuberculosis; 2) pregnant women; 3) patient with mental disorders, uncontrolled diabetes or severe cardiopulmonary disorders. Approval for this retrospective study was obtained from the Ethics committee of Army Medical Center, and all patients have signed the informed consent. This study is an extension of a previously reported work. ${ }^{12,13}$ This study was performed in accordance with the ethical standards of the 1964 Declaration of Helsinki and its most recent amendments.

Information on patient demographic characteristics and data on the extent of resection, adjuvant treatment, disease course, and survival were collected from our electronic medical records and through phone call follow-up. The extent of tumor resection was classified as total, subtotal $(<100 \%$ and $\geq 75 \%$ of gross total removal), or partial $(<75 \%$ of gross tumor removal) resection according to the integrative analysis of intraoperative impressions in surgery and postoperative MRI findings. ${ }^{4}$ Multiple lobes and multiple lesions were defined as involvement of two or more lobes connected by direct routes of spread and multiple sites not connected by obvious routes of spread. ${ }^{14}$ Progression-free survival (PFS) was defined as the time from diagnosis to tumor progression based on the Response Assessment in Neuro-Oncology (RANO) criteria $^{15,16}$ or the date of the last follow-up examination if the patient did not show disease progression. Overall survival (OS) was defined as the time from diagnosis to death or the date of the last follow-up examination if the patient did not die. Decisions on disease progression were made by the consensus of two neuroradiologists (H.-G and H.-Y.K., with 6 and 11 years of experience in brain MRI, 
Table I The Clinical Characteristics of Patients

\begin{tabular}{|c|c|c|c|}
\hline \multirow{2}{*}{$\begin{array}{l}\text { Characteristics/ } \\
\text { Variables }\end{array}$} & \multicolumn{2}{|c|}{ IDH I Mutation Status } & \multirow[t]{2}{*}{$P$ value } \\
\hline & $\begin{array}{l}\text { Mutated } \\
(n=43)\end{array}$ & $\begin{array}{l}\text { Wild } \\
(n=I 7)\end{array}$ & \\
\hline $\begin{array}{l}\text { Age (mean } \pm \text { SD, } \\
\text { years) }\end{array}$ & $41 \pm 10$ & $50 \pm 17$ & 0.059 \\
\hline $\begin{array}{l}\text { Gender } \\
\text { Male } \\
\text { Female }\end{array}$ & $\begin{array}{l}22(51.2 \%) \\
21(48.8 \%)\end{array}$ & $\begin{array}{l}8(47.06 \%) \\
9(52.94 \%)\end{array}$ & 1.000 \\
\hline $\begin{array}{l}\text { Location } \\
\text { Frontal lobe } \\
\text { Temporal lobe } \\
\text { Parietal lobe } \\
\text { Occipital lobe } \\
\text { Multiple lobes } \\
\text { Multiple lesions }\end{array}$ & $\begin{array}{l}17 \\
5 \\
1 \\
0 \\
18 \\
2\end{array}$ & $\begin{array}{l}2 \\
3 \\
1 \\
1 \\
10 \\
0\end{array}$ & $\begin{array}{l}\text { NA } \\
\text { NA } \\
\text { NA } \\
\text { NA } \\
\text { NA } \\
\text { NA }\end{array}$ \\
\hline $\begin{array}{l}\text { WHO grade } \\
\text { Grade II } \\
\text { Grade III }\end{array}$ & $\begin{array}{l}30(69.77 \%) \\
13(30.23 \%)\end{array}$ & $\begin{array}{l}7(41.18 \%) \\
10(58.82 \%)\end{array}$ & 0.075 \\
\hline $\begin{array}{l}\text { VSImax (mean } \\
\pm \mathrm{SD}, \mu \mathrm{m}) \\
\text { VSImean (mean } \\
\pm \mathrm{SD}, \mu \mathrm{m})\end{array}$ & $\begin{array}{l}106.45 \\
\pm 60.3 \\
74.06 \pm 44.7\end{array}$ & $\begin{array}{l}|55.7 \pm 55.6| \\
|| 3.4 \mid \pm 44.42\end{array}$ & $\begin{array}{l}0.005 \\
0.003\end{array}$ \\
\hline $\begin{array}{l}\text { Extent of resection } \\
\text { Gross total resection } \\
\text { Subtotal resection } \\
\text { Partial resection }\end{array}$ & $\begin{array}{l}25 \\
8 \\
10\end{array}$ & $\begin{array}{l}9 \\
5 \\
3\end{array}$ & $\begin{array}{l}\text { NA } \\
\text { NA } \\
\text { NA }\end{array}$ \\
\hline $\begin{array}{l}\text { Histopathology } \\
\text { Diffuse astrocytoma } \\
\text { Oligodendroglioma } \\
\text { Oligoastrocytoma } \\
\text { Anaplastic astrocytoma } \\
\text { Anaplastic } \\
\text { oligodendroglioma } \\
\text { Anaplastic } \\
\text { oligoastrocytoma }\end{array}$ & $\begin{array}{l}12 \\
11 \\
7 \\
1 \\
7 \\
5\end{array}$ & $\begin{array}{l}4 \\
3 \\
0 \\
6 \\
2 \\
2\end{array}$ & $\begin{array}{l}\text { NA } \\
\text { NA } \\
\text { NA } \\
\text { NA } \\
\text { NA } \\
\text { NA }\end{array}$ \\
\hline $\begin{array}{l}\text { I p/ / 9q } \\
\text { co-deletion } \\
\text { intact }\end{array}$ & $\begin{array}{l}28(65.12 \%) \\
15(34.88 \%)\end{array}$ & $\begin{array}{l}5(29.41 \%) \\
12(70.59 \%)\end{array}$ & 0.020 \\
\hline $\begin{array}{l}\text { Adjuvant therapy } \\
\text { Radiation } \\
\text { Chemotherapy } \\
\text { Chemoradiotherapy } \\
\text { Untreated } \\
\text { Unknown }\end{array}$ & $\begin{array}{l}4 \\
9 \\
21 \\
3 \\
6\end{array}$ & $\begin{array}{l}1 \\
4 \\
9 \\
3 \\
0\end{array}$ & $\begin{array}{l}\text { NA } \\
\text { NA } \\
\text { NA } \\
\text { NA } \\
\text { NA }\end{array}$ \\
\hline
\end{tabular}

Notes: Except where indicated, data are numbers of patients, and numbers in parentheses are percentage. Independent sample t-tests or Mann-Whitney U-tests, as appropriate, were used to compare the differences in continuous variables. The Chi-square testing was used to analyze the constituent ratio of variables. NA, not applicable. respectively) or based on the pathological diagnosis (if available). The data of patients without an event were censored at the date of the most recent follow-up, regardless of whether they were scheduled for future follow-ups or had been lost to follow-up.

\section{IDH Mutation and Ip/I9q Co-Deletion Detection}

IDH mutation status was determined using polymerase chain reaction (PCR) and pyrosequencing. Detailed protocols of these methods have been described elsewhere. ${ }^{17}$ Briefly, genomic DNAs were isolated from paraffin-embedded tumor tissues with a QIAamp DNA Mini Kit (Qiagen) according to the manufacturer's protocol. Forward primer 5'CGGTCTTCAGAGAAGCCATT-3' and reverse primer 5'GCAAAATCACATTATTGCCAAC-3' for IDH1 were used for PCR amplification. Fluorescence in situ hybridization (FISH) was used to assess 1p/19q co-deletion.

\section{MRI Protocol}

Preoperative MRI was performed using a 3T scanner (Magnetom Verio; Siemens, Erlangen, Germany). The routine imaging protocol included the following sequences: sagittal T1-weighted spin-echo (TR/TE, $440 \mathrm{~ms} / 2.48 \mathrm{~ms}$ ), axial T2-weighted fast spin-echo (FSE) (TR/TE, 4900ms/ 96ms), axial T1-weighted spin-echo sequence (TR 250ms, TE $2.67 \mathrm{~ms}$ ) performed before and after intravenous administration of gadolinium-DTPA (Magnevist, BayerSchering), $5 \mathrm{~mm}$ slice thickness, $1 \mathrm{~mm}$ intersection gap. MRI data for vessel size imaging (VSI) was acquired using a $1.5 \mathrm{~T}$ system (GE Healthcare) with following sequences: i.v. injection of Gd-DTPA using a gradientecho spin-echo (GE-SE) sequence, with acquisition parameters: field of view $=24 \times 24 \mathrm{~cm}$, TR $1500 \mathrm{~ms}$, TE (GE) $30 \mathrm{~ms}$, TE (SE) $100 \mathrm{~ms}$, flip angles $=90^{\circ}$, matrix size $=64$ $\times 64$, NEX 1 , slice number was 7 , and 50 images were obtained at each slice. As well, $0.2 \mathrm{mmol}$ of gadobutrol per kilogram of body weight was injected at a rate of $3 \mathrm{~mL} / \mathrm{s}$ and immediately followed by a $20 \mathrm{~mL}$ bolus of saline.

\section{VSI Measurement}

VSI images were analysed using an Advantage Workstation (version 4.9; General Electric Medical Systems) equipped with a dedicated software package (VSI; GE Medical Systems). Detailed protocols for the post-acquisition image processing have been described elsewhere. ${ }^{11}$ The VSI image threshold was adjusted to 
$0-80 \mu \mathrm{m}$ or $0-120 \mu \mathrm{m}$. The MR T2-weighted original image was used as a guide for tumor location. A handdrawn ROI corresponding to the hot-spot area avoiding any blood vessels and necrotic parts in the tumor tissue on the VSI map was chosen for the calculation of VSI values. Circular ROIs of $50-60 \mathrm{~mm}^{2}$ were placed on the tumoral parenchyma to yield quantitative data, and the circular ROIs were obtained from at least five adjacent regions. The average and max of the obtained values were named the VSImean values and VSImax values, respectively. Two experienced neuroradiologists (H.-Y.K. and H.-G, with 11 and 6 years of experience, respectively) were blinded to the histopathologic diagnosis and completed measurements, independently.

\section{Immunohistology Analysis}

An immunohistology kit (Zhong Shan-Golden Bridge Biological Technology company, Beijing, China) was used to detect CD34 expression in the tumor tissues embedded using paraffins according to the manufacturer's instruction. The results were observed using a microscope (Olympus BX41, France) and then analysed using CellSens Standard software. The microvascular diameter was calculated from at least five fields of the largest diameter using Image-Pro Plus 5.0 software. The measurements were performed by an experienced pathologist (H.L.X, with $>20$ years of experience) who was blinded to the MRI results.

\section{Statistical Analysis}

All results are expressed as the mean $\pm \mathrm{SD}$ or median \pm $10-90$ percentile. The $P$ value of less than 0.05 is considered statistically significant. Inter-observer reliability of VSI value measurements between the two radiologists was evaluated by intraclass correlation coefficients (ICC); ICC > 0.75 indicated excellent agreement. Independent-samples $T$-test or Mann-Whitney $U$-test was used to analyse the differences in VSI values between patients with IDHmutant glioma and those with IDH wild-type glioma and between patients with grade II glioma and those with grade III glioma. The Chi-square testing was used to analyze the constituent ratio of variables. Pearson correlation analysis was used to evaluate the relationship between VSI values and histological microvascular diameters. A receiver operating characteristic (ROC) curve was used to assess the specificity and sensitivity of VSImean and VSImax in distinguishing IDH-mutated LGGs from IDH wild-type LGGs, and the optimal cutoff values were determined using the
Youden index. Logistic regression was used to determine the ability of variables to discriminate IDH mutational status. The PFS and OS (4 years) of patients were analysed using Kaplan-Meier curves and the Log-rank test. For PFS, Cox regression analysis was employed to assess the risk factors, including gender, age, tumor location, adjuvant therapy, extent of resection, histopathology type, IDH mutation status, $1 \mathrm{p} / 19 \mathrm{q}$ co-deletion and VSI values. GraphPad Prism 8.0.1 and R (version 3.6.2) software were used for statistical analysis.

\section{Result}

\section{VSI Values Correlate with Microvessel Diameters in LGGs}

There was an excellent interobserver agreement for the VSImean (ICC $=0.955)$ and VSImax $(\mathrm{ICC}=0.923)$ measurements, and the VSImax and VSImean values were calculated based on the average values of the two observers. To investigate whether VSI values are associated with microvessel diameters in LGGs, we obtained VSImax and VSImean values and microvessel diameters in 60 LGG samples. We found that both VSImax and VSImean values significantly correlated with microvessel diameters in LGGs (Figure 1A and B), suggesting that VSI and histopathological approaches have a high consistency in evaluating the microvessel size in LGGs.

\section{VSI Values are Lower in IDH-Mutant LGGs and in Grade II Gliomas}

Next, we sought to analyse the association of VSI values with IDH mutation status and glioma grading. We found that both VSImax and VSImean values were significantly lower in IDH-mutant LGGs than in IDH wild-type LGGs (Figure 2A and E). Moreover, the VSImax and VSImean values were also significantly lower in grade II gliomas with IDH mutation than in those with IDH wild type (Figure 2B and F), whereas in grade III gliomas, there were no significant differences between IDH mutation and IDH wild type (Figure 2C and G). Furthermore, we also found that the VSImax and VSImean values were significantly lower in grade II gliomas than in grade III gliomas (Figure 2D and H). The representative images of MRI and pathology are shown in Figure 3. These results suggest that the VSImax and VSImean values correlate with IDH mutation status and glioma grading. 

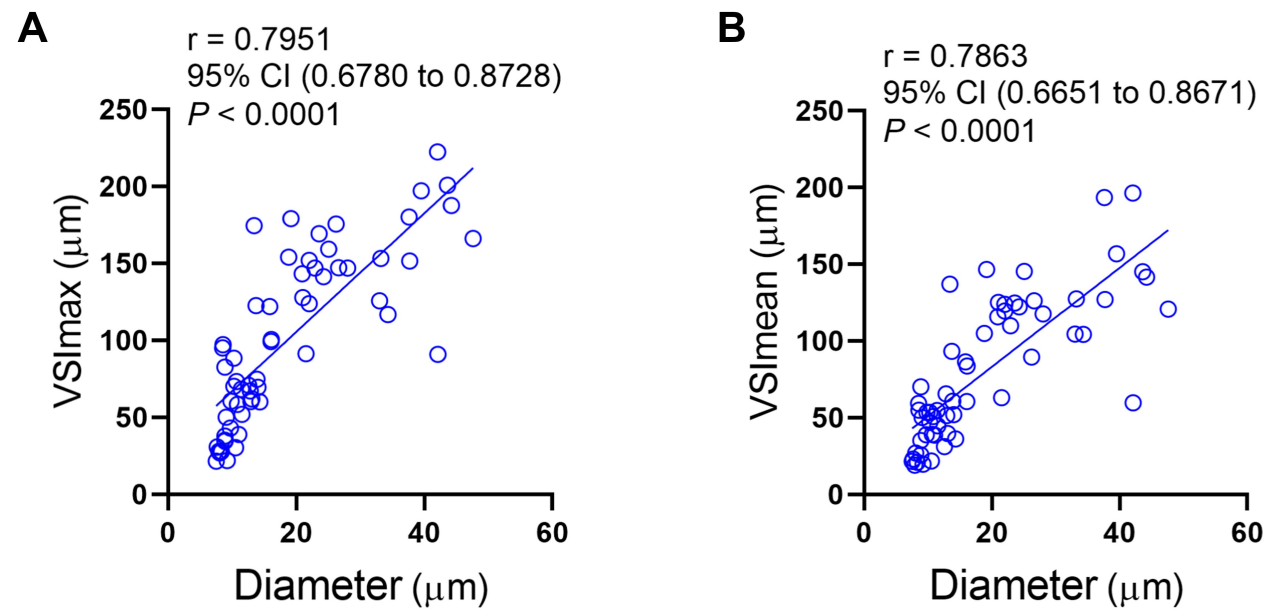

Figure I The VSI values correlate with microvessel diameter in LGGs. (A) VSImax and (B) VSImean values positively correlate with microvessel diameter in 60 LGG patients.

A

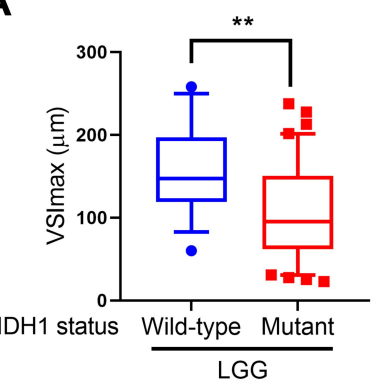

E

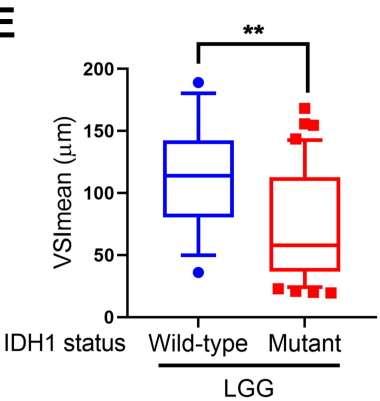

B

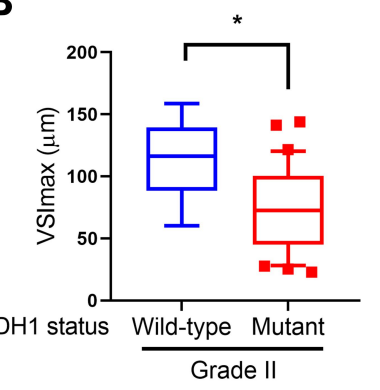

$\mathbf{F}$

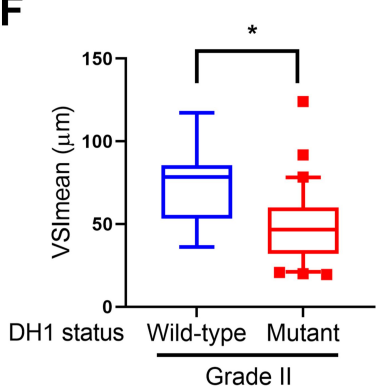

C

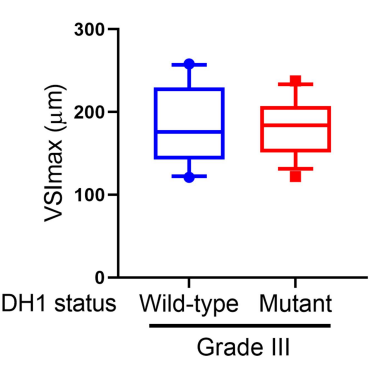

G

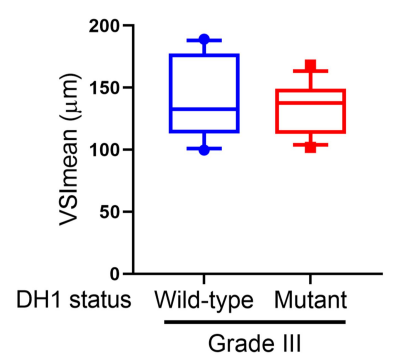

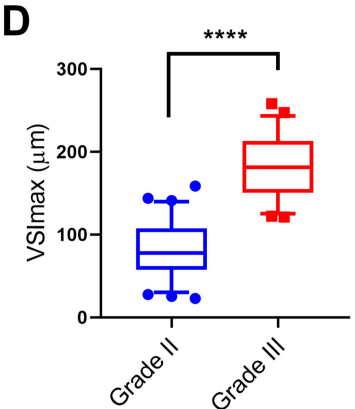

H

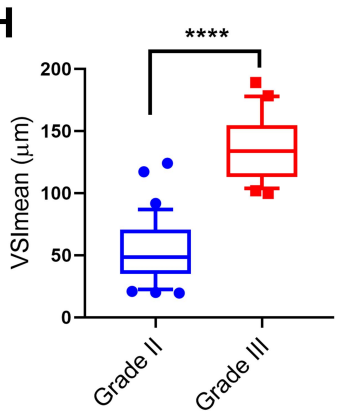

Figure 2 The VSI values are lower in IDH-mutant LGGs and in grade II gliomas. (A and E) IDH wild-type LGGs $(n=I 7)$ and IDH-mutant LGGs ( $=43$ ). (B and F) Grade II gliomas with IDH wild type $(n=7)$ and grade II gliomas with IDH mutation $(n=20)$. (C and $\mathbf{G})$ Grade III gliomas with IDH wild type $(n=I 0)$ and grade III gliomas with IDH mutation $(n=13)$. (D and $\mathbf{H})$ Grade II gliomas $(n=37)$ and grade III gliomas $(n=23)$. Boxes show the VSI values $(\mu \mathrm{m})$ of different types of LGGs, with appearance: box and whiskers, and whiskers: $10-90$ percentile. $* P<0.05 ; * * P<0.01$; $* * * * P<0.000$ I.

\section{VSI Values May Serve as a Marker for the Prediction of IDH Mutation Status in LGGs}

Then, we analysed the potential power of the VSImax and VSImean values as markers in differentiating IDH-mutant LGGs from IDH wild-type LGGs. ROC curve analyses revealed that VSImax had an AUC value of 0.7305 in distinguishing IDH-mutant LGGs from IDH wild-type LGGs, with $62.79 \%$ sensitivity and $82.35 \%$ specificity when an optimal cutoff value of $112.8 \mu \mathrm{m}$ was used (Figure 4A), while VSImean had an AUC value of 0.7401 with $65.12 \%$ sensitivity and $82.35 \%$ specificity when an optimal cutoff value of $78.5 \mu \mathrm{m}$ was used (Figure 4B). We sought to combine the VSImean values with other factors including the VSImax values, age and tumor location using a stepwise logistic regression method, and a formula (IDH-mutant signature = $-5.42125+$ VSImean $\times 0.01550+$ tumor location $\times$ 

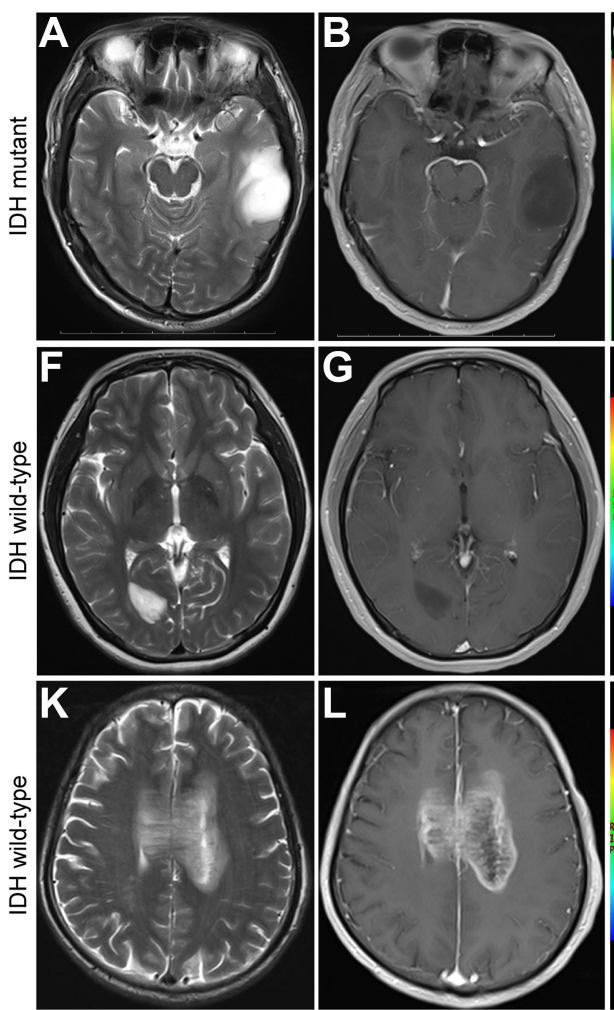
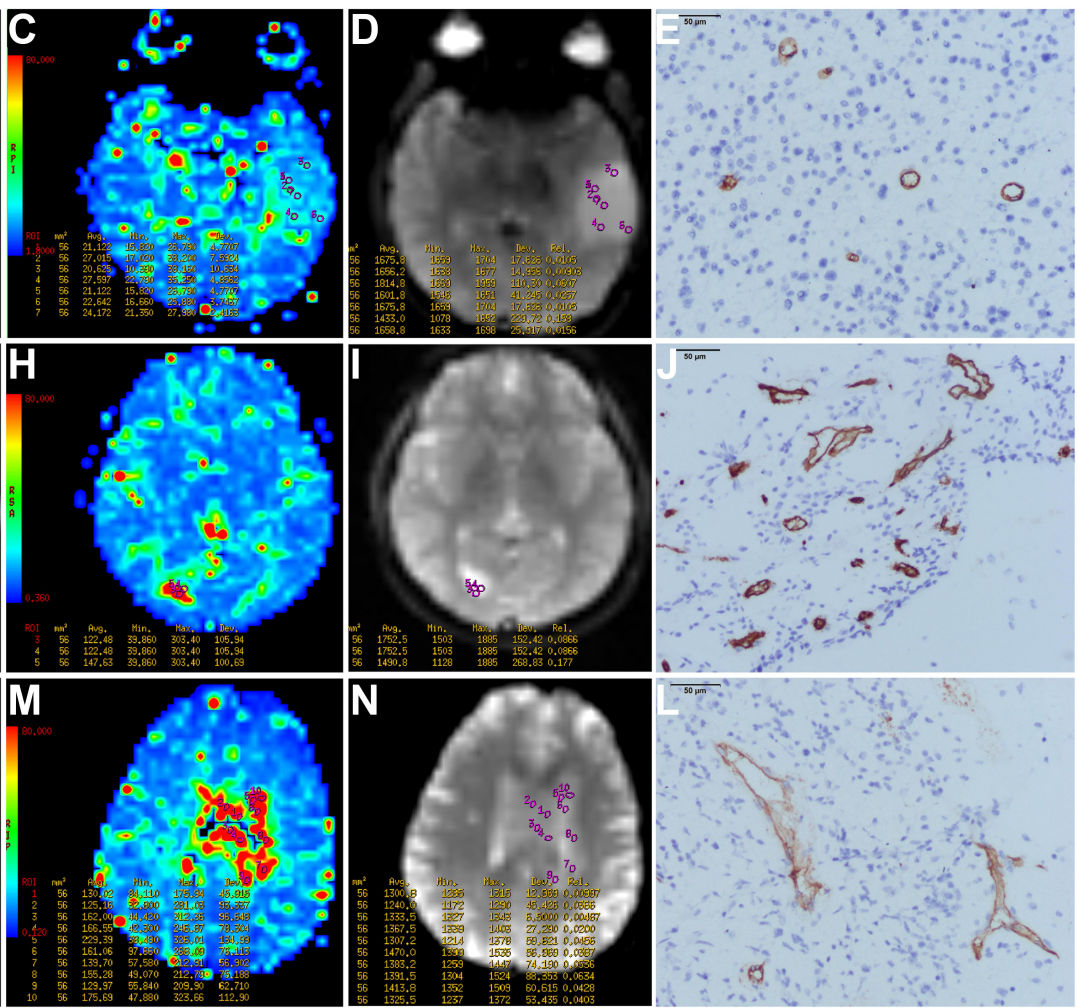

Figure $3 \mathrm{MRI}$, VSI map and CD34 staining of IDH-mutant and wild-type LGGs. A 3 I-year-old man with an IDH-mutant diffuse astrocytoma (WHO grade II) at the left temporal lobe showing homogeneous high signal intensity on a T2-weighted image (A) and no enhancement on a contrast-enhanced TI-weighted image (B). ROls were drawn on the area showing the highest signal within the lesion on the processed VSI map image (C) and a corresponding raw VSI image (D). Representative photograph of CD34 staining for the corresponding tumor micro-vessel (E). The VSImean is $23.47 \mu \mathrm{m}$ and VSImax is $27.597 \mu \mathrm{m}$, which belongs to the VSI-low group according to the optimized cutoff values for OS and PFS prediction. The PFS of this patient was more than 60 months. This patient was alive at the last follow-up. A I9-year-old woman with an IDH wild-type diffuse astrocytoma (WHO grade II) at the right occipital lobe showing similar image features on a T2-weighted image (F) and a contrast-enhanced TIweighted image $(\mathbf{G})$ as the IDH-mutant diffuse astrocytoma case. ROls were drawn on the area that showed the highest signal on the processed VSI map image $(\mathbf{H})$ and a raw VSI image (I). Representative photograph of CD34 staining for the corresponding tumor micro-vessel (J). The VSImax value is I47.63 $\mu \mathrm{m}$ and the VSImean value is $82.26 \mu \mathrm{m}$, which belongs to the VSI-high group according to the optimized cutoff values for OS and PFS prediction and is close to cutoff values for OS and PFS prediction. The PFS of this patient was more than 46 months. They both underwent gross total resection and chemoradiotherapy after surgery. A 72 -year-old man with an IDH wildtype anaplastic astrocytoma (WHO grade III) at the body of the corpus callosum and cingulate gyrus showing heterogeneous high signal intensity on a T2-weighted image (K) and intense but inhomogeneous enhancement on a contrast-enhanced TI-weighted image (L). ROls were drawn on the area that showed the highest signal on the processed VSI map image (M) and a raw VSI image (N). Representative photograph of CD34 staining for the corresponding tumor micro-vessel (L). The VSImax value is $229.39 \mu \mathrm{m}$ and VSImean value is $156.7 \mu \mathrm{m}$, which belongs to the VSI-high group according to the optimized cutoff values for OS and PFS prediction. He underwent a subtotal resection and without any therapy after surgery. The OS of this patient was only 12 months.

A IDH1 wild-type vs. mutant LGG (VSImax)

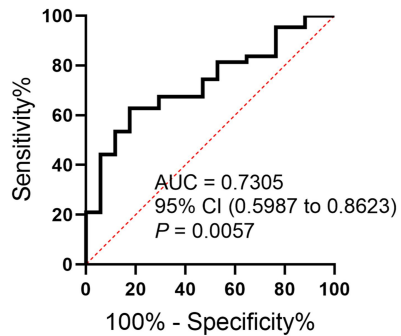

B

IDH1 wild-type vs. mutant LGG (VSImean)

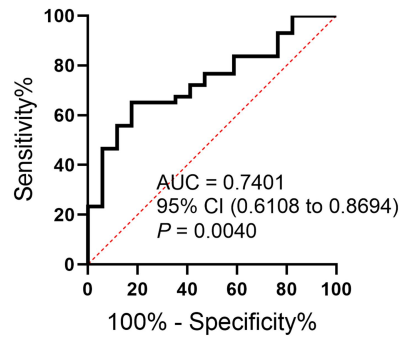

C

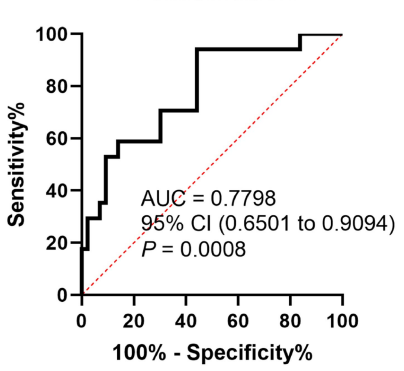

D

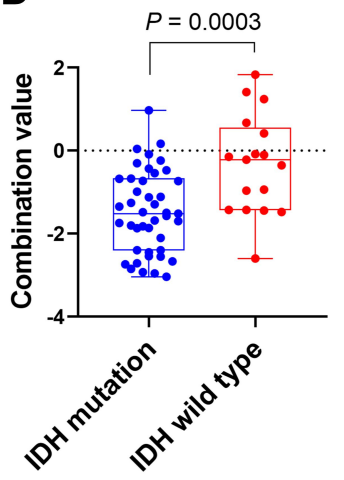

Figure 4 VSI values may serve as a marker for the prediction of IDH mutation status in LGGs. ROC curves were established. IDH wild-type LGG cases ( $\mathrm{n}=$ I7) versus those IDH-mutated LGG cases $(\mathrm{n}=43)$. (A) ROC curve: VSI max. (B) ROC curve: VSImean. (C) Combination of the VSImean values with tumor location and age using stepwise logistic regression. (D) Combination value between IDH-mutant LGGs $(n=43)$ and IDH wild-type LGGs $(n=17)$. 
$0.85926+$ age $\times 0.03618)$ was obtained. Then, we used this formula to integrate the three factors and obtained an AUC value of 0.7798 to distinguish IDH mutation and IDH wild type, with $94.12 \%$ sensitivity and $55.81 \%$ specificity (Figure 4C and D). However, the VSImax values were not included in this formula, possibly due to their insufficient effect. These results suggest that the VSImean values and integrated factors have the potential power to predict IDH mutation status in LGGs.

\section{VSI Values are Associated with the PFS and OS of Patients with LGGs}

Next, we sought to analyse whether the VSI values correlate with the PFS and OS of patients with LGGs. The follow-ups of 60 patients with LGGs include: 32 of 60 patients had tumor progression (10 of 32 patients with IDH wild-type LGGs and 22 of 32 patients with IDHmutant LGGs); 16 patients died (7 of 17 patients with IDH wild-type LGGs and 9 of 47 patients with IDHmutant LGGs); and 9 of 60 patients were lost to followup. The median PFS of the patients was 42.0 months. As the survival probability did not fall below 0.5 , the median OS was not calculated. The mean OS of the patients was 61.279 months. We then divided the patients into different groups for analysis at four-year follow-up based on corresponding cutoff values (VSImax-Low versus VSImaxHigh and VSImean-Low versus VSImean-High), IDH mutation status (IDH wild-type versus IDH mutant) and glioma grading (grade II versus grade III). The mean OS of the VSImax-High group (36.515 months; 95\% CI, 30.415-42.615) and VSImean-High group (35.970 months; 95\% CI, 29.683-42.258) was significantly shorter than the VSImax-Low group (44.464 months; 95\% CI, 40.563-48.365) and VSImean-Low group (44.604 months; 95\% CI, 40.847-48.362) (Figure 5A and B). And the mean PFS of the VSImax-High group (27.437 months; 95\% CI, 20.936-33.938) and VSImean-High group (26.707 months; 95\% CI, 20.089-33.325) was significantly shorter than the VSImax-Low (38.962 months; 95\% CI, 33.65544.268) and VSImean-Low groups (39.178 months; 95\% CI, 34.053-44.303) (Figure 5C and D). The representative images are shown in Figure 3. The mean OS and PFS of the IDH wild-type group were also significantly shorter than that of the IDH-mutant group (Figure S1A and D). In addition, the mean OS and median PFS of the grade III group were both shorter than those of the grade II group (Figure S1B and $\underline{E}$ ), but they were not significantly different between IDH wild-type grade II and grade III groups (Figure S1C and $\underline{F}$ ).

\section{IDH Mutation Status, VSImean, and} Multiple Lesions or Lobes are Risk Factors for PFS in Patients with LGGs

Next, we sought to analyse the risk factors including the VSI values, IDH status, $1 \mathrm{p} / 19 \mathrm{q}$ co-deletion status and multiple clinical characteristics for PFS in patients with LGGs. By performing univariate Cox analysis, we found that IDH mutation status, VSImax values, VSImean values, grade, age, adjuvant therapy, and multiple lesions or lobes were associated with PFS, but gender, histopathology type, $1 \mathrm{p} / 19 \mathrm{q}$ co-deletion status and extent of resection were not correlated with PFS (Table 2). Furthermore, according to the results of univariate analysis, four models for predicting PFS were generated using multivariate Cox regression analysis. We found that the factors of multiple lesions or lobes, IDH mutation status and VSImean values were hazard factors for PFS in different models (Table 3).

\section{Discussion}

In this study, we found that the VSImax and VSImean values are lower in IDH-mutant LGGs than in IDH wildtype LGGs and in grade II gliomas than in grade III gliomas; ROC curve analyses suggested that VSI values may serve as a potential marker for distinguishing IDHmutant LGGs from IDH wild-type LGGs; and the combination of VSImean values, age and tumor location showed more power to predict IDH genotype in LGGs. Furthermore, we demonstrated that LGG patients with high VSI values have worse survival rates than those with low VSI values at the four-year follow-up for PFS and OS. Moreover, univariate Cox analysis revealed that VSI values, IDH mutation status, grade, age, adjuvant therapy, and multiple lesions or lobes were risk factors for the survival of LGG patients. Multivariate Cox proportional hazards regression analysis suggested that IDH mutation status, VSImean values and multiple lesions or lobes might be independent risk factors for PFS of LGG patients. These suggested that the VSImean values may be potential imaging features that can be used to detect IDH mutation status and patient survival in LGGs.

There are differences in angiogenesis between IDH wild-type and mutant gliomas. ${ }^{1,10}$ Studies have indicated that IDH wild-type LGGs exhibit high enrichment of 
A

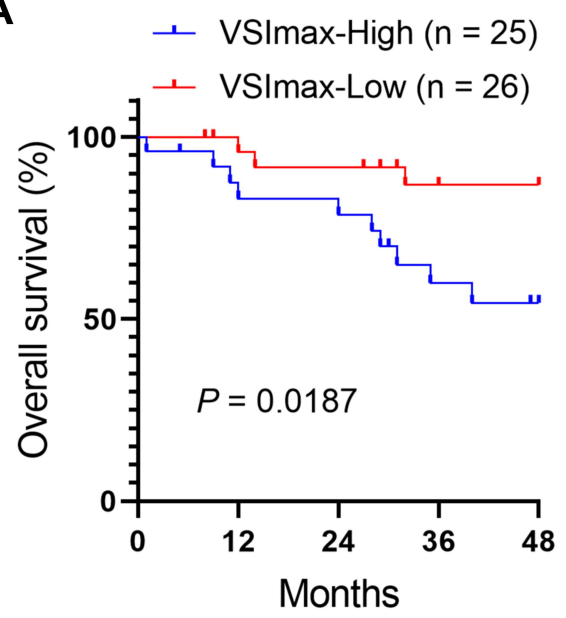

C

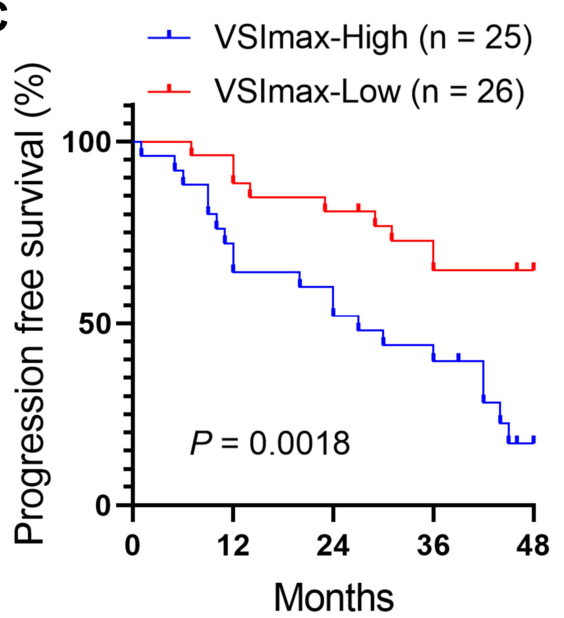

B

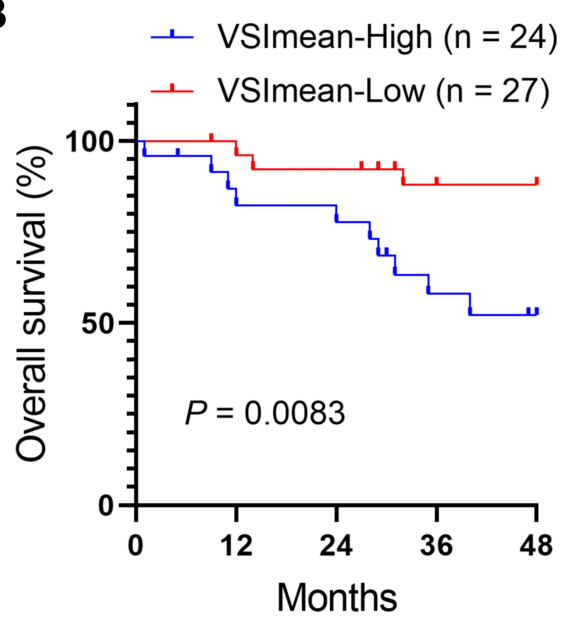

D

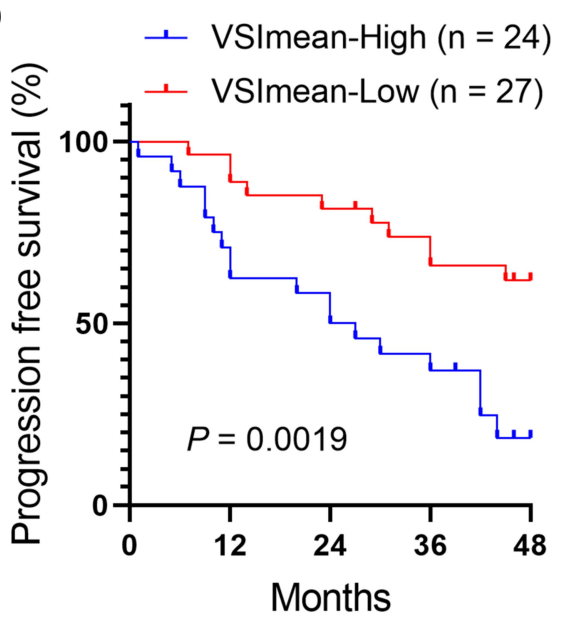

Figure 5 VSI values are associated with the PFS and OS of patients with LGGs at the four-year follow-up. Kaplan-Meier curve survival analyses show the OS and PFS of patients with different types of LGGs. (A and $\mathbf{C}$ ) The patients were divided into two groups of VSImax-High and VSImax-Low, based on a VSImax cutoff value of I I 2.8 $\mu$; (B and D) the patients were divided into two groups of VSImean-High and VSImean-Low, based on VSImean cutoff value of 78.5 $\mu \mathrm{m}$.

anoxia- and angiogenesis-associated genes and an increased pericyte coverage of micro-vessels compared with IDHmutant LGGs. ${ }^{18,19}$ The variation of glioma angiogenesis can be differentiated using a dynamic susceptibility contrast (DSC) perfusion MRI model, ${ }^{1,10}$ where $\mathrm{rCBV}$ is a representative parameter that is widely used. ${ }^{1,20,21}$ However, another relatively new parameter, VSI, has rarely been reported. VSI can describe the structural heterogeneity of the brain microvasculature as well as the microvascular oxygen content and spatial arrangement. ${ }^{11}$ We also found that VSI values are positively correlated with microvessel diameter. VSI was calculated based on curves of the GE relaxation rate $\mathrm{R} 2 *$ and $\mathrm{SE}$ relaxation rate $\mathrm{R} 2$ in the signal drop portion. ${ }^{22}$ Choi et al found that using representative T2* susceptibility signal intensity-time curves from DSC perfusion MRI to distinguish the IDH genotype will obtain a better effect than rCBV because these curves have different upslopes in the signal drop portion. ${ }^{7}$ In this study, our findings revealed that VSI values act as a marker to distinguish IDH wild-type LGGs from IDHmutant LGGs, suggesting that VSI may be an alternative method for the evaluation of IDH mutation status in LGGs. Our previous study indicated that VSI is more accurate than rCBV in grading glioma. ${ }^{12}$ However, we did not compare the effect of VSI with $\mathrm{rCBV}$ on the evaluation of IDH mutation status in the present study; therefore, it is necessary to evaluate which one is better in the future. In addition, using stepwise logistic regression, we found that the VSImean values, age and multiple lesions or lobes were independent variables for identifying the IDH genotype, and their combination had a higher AUC. In line with our study, Villanueva-Meyer et al also showed multifocal tumor and age were independently associated with IDH mutation status. $^{14}$ 
Table 2 Univariate Survival Analysis

\begin{tabular}{|l|l|l|l|}
\hline Features & $\begin{array}{l}\text { Hazard } \\
\text { Ratio }\end{array}$ & $\begin{array}{l}\text { 95\% Confidential } \\
\text { Interval }\end{array}$ & $P$ value \\
\hline VSImean & 1.011 & $1.004,1.019$ & 0.0029 \\
VSImax & 1.009 & $1.003,1.015$ & 0.0033 \\
IDH mutation & 0.3103 & $0.1409,0.6836$ & 0.0037 \\
Grade & 3.073 & $1.432,6.593$ & 0.0040 \\
Multiple lesions or lobes & 2.659 & $1.364,5.184$ & 0.0041 \\
Age & 1.036 & $1.004,1.068$ & 0.0254 \\
Adjuvant therapy after & 0.6957 & $0.4847,0.9987$ & 0.0492 \\
surgery & & & 0.4360 \\
Gender & 1.32 & $0.6563,2.653$ & 0.4620 \\
Ip/I9q co-deletion & 0.7687 & $0.3812,1.55$ & 0.4900 \\
Histopathology & 0.8642 & $0.5708,1.308$ & 0.8890 \\
Extent of resection & 1.031 & $0.6732,1.579$ & \\
\hline
\end{tabular}

It is well known that patients with IDH-mutant LGGs have better outcomes than those with IDH wild-type LGGs, ${ }^{6-8}$ and grade III glioma leads to poorer survival rates compared with grade II glioma, but the survival time is not significantly different between grade II and grade III IDH wild-type gliomas. ${ }^{5}$ Our study also confirmed the same results using Kaplan-Meier survival analysis and Cox proportional hazards models, and we found that IDH mutation status rather than grade was an independent prognostic factor for PFS. Interestingly, we are the first to observe that LGG patients with high pre-treatment VSI values had worse survival rates, both PFS and OS. A few studies have shown that vascular normalization can improve tumor perfusion and thereby increase oxygenation, which can improve the OS of patients with glioblastoma, and these patients with vascular normalization after cediranib, which can be evaluated by VSI, also have longer OS and PFS. ${ }^{17,23}$ Our findings suggest that the pretreatment VSI value is a predictive factor for the outcome of LGG patients, and it does not depend on the treatment strategy or vessel change after treatment. Furthermore, we found that VSImean values had a better effect than VSImax values in predicting LGG patient outcomes. The underlying reason may be that the VSImean values were built from multiple values in hot-spot areas, so they can reflect the integrated status of glioma, but the VSImax values were obtained from the highest value, which more easily results in a bias.

Our analyses of univariate Cox regression indicated that, except for the VSI values and IDH mutation status, several clinical features were correlated with LGG patient outcomes, including older age, postoperative adjuvant chemoradiotherapy, and multiple lesions or lobes, and a large number of studies support our findings. ${ }^{24-26}$ For multivariate Cox regression, we established four models based on different risk factors obtained from the results of univariate analysis. Each model contains three factors at most, due to the limit on the number of our cases. We found that the VSImean values rather than the VSImax values may be an independent risk factor for LGG patients. We further found that the VSImean values and IDH mutation status are two independent risk factors when we combine each of the two factors with two other clinical factors. These results suggested that when VSI serves as a risk factor, its efficiency is similar to that of IDH for predicting LGG patient outcomes. Previous studies have indicated that the pre-treatment $\mathrm{rCBV}$ value is also an independent prognostic factor of glioma, and decreased rCBV values correlate with longer OS. ${ }^{21,27-29}$ VSI and rCBV were two different parameters in perfusion imaging that reflect different hemodynamic changes and microvascular structures, and their comparison with regard to the OS or PFS of patients with LGGs needs to be explored in the future. However, factors including extent of resection, $1 \mathrm{p} / 19 \mathrm{q}$ co-deletion status and histopathology type were not associated with PFS in our study, although these factors may be prognostic factors for glioma. ${ }^{30,31}$ Several studies obtained the same results as our study. For instance, Bio Joo et al indicated

Table 3 Multivariate Prognostic Models, Clinical Factors with IDH or VSI Values, for Predicting PFS

\begin{tabular}{|c|c|c|c|c|c|c|c|c|}
\hline \multirow[t]{2}{*}{ Features } & \multicolumn{2}{|l|}{ Model I } & \multicolumn{2}{|l|}{ Model 2} & \multicolumn{2}{|l|}{ Model 3} & \multicolumn{2}{|l|}{ Model 4} \\
\hline & HR(95\% Cl) & $P$ value & HR(95\% Cl) & $P$ value & HR(95\% Cl) & $P$ value & HR(95\% Cl) & $P$ value \\
\hline IDH & $0.4103(0.1658,1.015)$ & 0.05397 & & & $0.3548(0.1552,0.8111)$ & 0.0140 & & \\
\hline VSImean & & & $1.0013(0.9864,1.017)$ & 0.85896 & & & $1.0095(1.0015,1.018)$ & 0.0202 \\
\hline Grade & $2.1883(0.9146,5.235)$ & 0.07850 & $2.7060(0.5777,12.674)$ & 0.20638 & & & & \\
\hline$M L$ & $2.8187(1.3947,5.697)$ & 0.00389 & $2.6494(1.2996,5.401)$ & 0.00734 & & & & \\
\hline Age & & & & & I.0243 (0.9954, I.542) & 0.1006 & $1.0220(0.9929,1.052)$ & 0.1401 \\
\hline Therapy & & & & & $0.7156(0.4944,1.0358)$ & 0.0761 & $0.7523(0.5264,1.075)$ & 0.1185 \\
\hline
\end{tabular}

Notes: HR, hazard ratio; $95 \% \mathrm{Cl}, 95 \%$ confidential interval; IDH, IDH mutation; ML, multiple lesions or lobes; Therapy, adjuvant therapy after surgery. 
that extent of resection was not related to PFS and OS in high-grade glioma; ${ }^{4}$ another study showed that there was no difference in either PFS or OS among the various histologies in WHO grade II gliomas; ${ }^{32}$ VillanuevaMeyer et al indicated that the $1 \mathrm{p} / 19 \mathrm{q}$ co-deletion is associated with the prognosis of patients with grade II gliomas, but there were no IDH wild-type tumors with $1 \mathrm{p} / 19 \mathrm{q}$ codeletion among their cases. ${ }^{14}$ Different from those cases, our patients have IDH wild-type LGG with 1p/19q codeletion, and Hameed et al revealed that $1 p / 19 q$ codeletion was not a significant predictor of OS in LGGs. ${ }^{33}$

Nevertheless, our study has several limitations. First, we used retrospective data, our sample size was relatively small. Due to the loss of follow-up for some patients, there were a lot of censored data, which affected the prognosis analysis, especially the accuracy of the judgment of overall survival, and most patients with IDH-mutant LGGs were still alive when the follow-up had been finished, so we did not conduct Cox regression analysis of OS. Second, we manually measured the VSI values, which will be influenced by some subjective factors, but because we used the same threshold to observe the imaging and the VSI values are absolute parameters, there was high consistency between different observers, suggesting that this method has acceptable repeatability. A previous study indicated that there was a slightly better correlation of Automatable Gaussian Normalized CBV with the OS of glioma patients than standard white matter normalized CBV, but the difference was not statistically significant. ${ }^{31}$ Therefore, we still obtain a good conclusion by using the hot-spot method, but more objective measurement methods still need further study.

In conclusion, our findings indicated that VSI values may serve as a predictor of IDH genotype in LGGs and of the prognosis of LGG patients.

\section{Funding}

The present study was supporting by the Chongqing Health Commission Fund (grant no. 2018QNXM026) and National Natural Science Foundation of China (grant no. 81871421) and National Key Research and Development Program of China (grant no. 2018YFC0115004).

\section{Disclosure}

The authors declare no conflicts of interest for this work.

\section{References}

1. Xing Z, Yang X, She D, Lin Y, Zhang Y, Cao D. Noninvasive assessment of IDH mutational status in World Health Organization grade II and III astrocytomas using DWI and DSC-PWI combined with conventional MR imaging. AJNR Am J Neuroradiol. 2017;38 (6):1138-1144. doi:10.3174/ajnr.A5171

2. Patel SH, Poisson LM, Brat DJ, et al. T2-FLAIR mismatch, an imaging biomarker for IDH and $1 \mathrm{p} / 19 \mathrm{q}$ status in lower-grade gliomas: a TCGA/TCIA project. Clin Cancer Res. 2017;23 (20):6078-6085. doi:10.1158/1078-0432.CCR-17-0560

3. Wu F, Wang ZL, Wang KY, et al. Classification of diffuse lower-grade glioma based on immunological profiling. Molecular Oncol. 2020;14(9):2081.

4. Joo B, Han K, Ahn SS, et al. Amide proton transfer imaging might predict survival and IDH mutation status in high-grade glioma. Eur Radiol. 2019;29(12):6643-6652. doi:10.1007/s00330-019-06203-x

5. Olar A, Wani KM, Alfaro-Munoz KD, et al. IDH mutation status and role of WHO grade and mitotic index in overall survival in grade II-III diffuse gliomas. Acta Neuropathol. 2015;129(4):585-596. doi:10.1007/s00401-015-1398-z

6. Han S, Liu Y, Cai SJ, et al. IDH mutation in glioma: molecular mechanisms and potential therapeutic targets. $\mathrm{Br} J$ Cancer. 2020;122(11):1580-1589. doi:10.1038/s41416-020-0814-x

7. Choi KS, Choi SH, Jeong B. Prediction of IDH genotype in gliomas with dynamic susceptibility contrast perfusion MR imaging using an explainable recurrent neural network. Neuro Oncol. 2019;21 (9):1197-1209.

8. Suh CH, Kim HS, Jung SC, Choi CG, Kim SJ. Imaging prediction of isocitrate dehydrogenase (IDH) mutation in patients with glioma: a systemic review and meta-analysis. Eur Radiol. 2019;29(2):745758. doi:10.1007/s00330-018-5608-7

9. Yoo RE, Yun TJ, Hwang I, et al. Arterial spin labeling perfusion-weighted imaging aids in prediction of molecular biomarkers and survival in glioblastomas. Eur Radiol. 2020;30 (2):1202-1211. doi:10.1007/s00330-019-06379-2

10. Leu K, Ott GA, Lai A, et al. Perfusion and diffusion MRI signatures in histologic and genetic subtypes of WHO grade II-III diffuse gliomas. J Neurooncol. 2017;134(1):177-188. doi:10.1007/s11060017-2506-9

11. Emblem KE, Mouridsen K, Bjornerud A, et al. Vessel architectural imaging identifies cancer patient responders to anti-angiogenic therapy. Nat Med. 2013;19(9):1178-1183. doi:10.1038/nm.3289

12. Kang HY, Xiao HL, Chen JH, et al. Comparison of the effect of vessel size imaging and cerebral blood volume derived from perfusion MR imaging on glioma grading. AJNR Am J Neuroradiol. 2016;37(1):51-57. doi:10.3174/ajnr.A4477

13. Guo H, Kang $\mathrm{H}$, Tong $\mathrm{H}$, et al. Microvascular characteristics of lower-grade diffuse gliomas: investigating vessel size imaging for differentiating grades and subtypes. Eur Radiol. 2019;29(4):18931902. doi:10.1007/s00330-018-5738-y

14. Villanueva-Meyer JE, Wood MD, Choi BS, et al. MRI features and IDH mutational status of grade II diffuse gliomas: impact on diagnosis and prognosis. AJR Am J Roentgenol. 2018;210(3):621-628. doi:10.2214/AJR.17.18457

15. Wen PY, Macdonald DR, Reardon DA, et al. Updated response assessment criteria for high-grade gliomas: response assessment in neuro-oncology working group. $J$ Clin Oncol. 2010;28 (11):1963-1972. doi:10.1200/JCO.2009.26.3541

16. van den Bent MJ, Wefel JS, Schiff D, et al. Response assessment in neuro-oncology (a report of the RANO group): assessment of outcome in trials of diffuse low-grade gliomas. Lancet Oncol. 2011;12 (6):583-593. doi:10.1016/S1470-2045(11)70057-2

17. Kong Z, Yan $\mathrm{C}$, Zhu R, et al. Imaging biomarkers guided anti-angiogenic therapy for malignant gliomas. Neuroimage Clin. 2018;20:51-60. doi:10.1016/j.nicl.2018.07.001 
18. Zhang L, He L, Lugano R, et al. IDH mutation status is associated with distinct vascular gene expression signatures in lower-grade gliomas. Neuro Oncol. 2018;20(11):1505-1516. doi:10.1093/ neuonc/noy088

19. Sun C, Zhao Y, Shi J, et al. Isocitrate dehydrogenase1 mutation reduces the pericyte coverage of microvessels in astrocytic tumours J Neurooncol. 2019;143(2):187-196. doi:10.1007/s11060-019-03156-5

20. Kickingereder P, Sahm F, Radbruch A, et al. IDH mutation status is associated with a distinct hypoxia/angiogenesis transcriptome signature which is non-invasively predictable with rCBV imaging in human glioma. Sci Rep. 2015;5(1):16238. doi:10.1038/srep16238

21. Liu X, Mangla R, Tian W, et al. The preliminary radiogenomics association between MR perfusion imaging parameters and genomic biomarkers, and their predictive performance of overall survival in patients with glioblastoma. J Neurooncol. 2017;135(3):553-560. doi:10.1007/s11060-017-2602-x

22. Lemasson B, Valable S, Farion R, Krainik A, Rémy C, Barbier EL. In vivo imaging of vessel diameter, size, and density: a comparative study between MRI and histology. Magn Reson Med. 2013;69 (1):18-26. doi:10.1002/mrm.24218

23. Batchelor TT, Gerstner ER, Emblem KE. Improved tumor oxygenation and survival in glioblastoma patients who show increased blood perfusion after cediranib and chemoradiation. Proc Natl Acad Sci U S A. 2013;110(47):19059-19064. doi:10.1073/pnas.1318022110

24. Tsao-Wei DD, Hu J, Groshen SG, Chamberlain MC. Conditional survival of high-grade glioma in Los Angeles County during the year 1990-2000. J Neurooncol. 2012;110(1):145-152. doi:10.10 07/s11060-012-0949-6

25. Lamborn KR, Chang SM, Prados MD. Prognostic factors for survival of patients with glioblastoma: recursive partitioning analysis. Neuro Oncol. 2004;6(3):227-235. doi:10.1215/S1152851703000620
26. Paech D, Dreher C, Regnery S, et al. Relaxation-compensated amide proton transfer (APT) MRI signal intensity is associated with survival and progression in high-grade glioma patients. Eur Radiol. 2019;29 (9):4957-4967. doi:10.1007/s00330-019-06066-2

27. Kickingereder P, Wiestler B, Burth S. Relative cerebral blood volume is a potential predictive imaging biomarker of bevacizumab efficacy in recurrent glioblastoma. Neuro Oncol. 2015;17(8):1139-1147. doi:10.1093/neuonc/nov028

28. Bennett IE, Field KM, Hovens CM. Early perfusion MRI predicts survival outcome in patients with recurrent glioblastoma treated with bevacizumab and carboplatin. J Neurooncol. 2017;131(2):321-329.

29. Law M, Young RJ, Babb JS, et al. Gliomas: predicting time to progression or survival with cerebral blood volume measurements at dynamic susceptibility-weighted contrast-enhanced perfusion MR imaging. Radiology. 2008;247(2):490-498. doi:10.1148/radiol.247 2070898

30. Ye D, Ma S, Xiong Y, Guan KL. R-2-hydroxyglutarate as the key effector of IDH mutations promoting oncogenesis. Cancer Cell. 2013;23(3):274-276. doi:10.1016/j.ccr.2013.03.005

31. Anzalone N, Castellano A, Cadioli M, et al. Brain gliomas: multicenter standardized assessment of dynamic contrast-enhanced and dynamic susceptibility contrast MR images. Radiology. 2018;287 (3):933-943. doi:10.1148/radiol.2017170362

32. Leeper HE, Caron AA, Decker PA, Jenkins RB, Lachance DH, Giannini C. IDH mutation, $1 \mathrm{p} 19 \mathrm{q}$ codeletion and ATRX loss in WHO grade II gliomas. Oncotarget. 2015;6(30):30,295-30,305. doi:10.18632/oncotarget.4497

33. Hameed NUF, Qiu T, Zhuang D, et al. Transcortical insular glioma resection: clinical outcome and predictors. J Neurosurg. 2018;131 (3):706-716. doi:10.3171/2018.4.JNS18424

\section{Publish your work in this journal}

Cancer Management and Research is an international, peer-reviewed open access journal focusing on cancer research and the optimal use of preventative and integrated treatment interventions to achieve improved outcomes, enhanced survival and quality of life for the cancer patient.
The manuscript management system is completely online and includes a very quick and fair peer-review system, which is all easy to use. Visit http://www.dovepress.com/testimonials.php to read real quotes from published authors. 